\title{
EMPATÍA Y COMPONENTES DE LA EMPATÍA EN ALUMNOS DE OBSTETRICIA DE LA UNIVERSIDAD NORBERT WIENER, PERÚ
}

\author{
CLAUDIA ARISPE ALBURQUEQUE \\ Universidad Norbert Wiener
}

En 2016, entre otros proyectos ejecutados con el auspicio del Fondo Concursable de Investigación para Docentes de la Universidad Wiener, se concretó aquel sobre empatía en estudiantes de Obstetricia de la institución, diseñado y ejecutado por quien firma esta noticia. Fue trabajado desde categorías propuestas por el doctor Víctor Díaz, de la Universidad de San Sebastián, de Santiago de Chile, y forma parte de un interés compartido por decenas de universidades de la región sobre este elemento difícil de asir como es el de la empatía del profesional de salud con sus pacientes. El resultado de la investigación, que ha sido trabajado con el propio doctor Díaz, está presentándose a una revista especializada. Sin embargo, dada la importancia del tema para con los planes curriculares de la carrera y la profesión de salud, se ofrece en la revista de la Universidad Wiener un avance del mismo.

Evaluar la empatía y los componentes de la empatía en alumnos de Obstetricia de la Universidad Wiener fue una investigación que se realizó con el objetivo de conocer el grado de empatía del estudiante para comprender las experiencias y los sentimientos del paciente con respecto a su proceso de salud-enfermedad. Diversos autores demuestran que la presencia de empatía permite mejorar la relación terapéutica con el profesional de salud, e incrementa la habilidad para el diagnóstico y tratamiento, lo que constituye ventajas para el paciente y los profesionales de la salud.

Por lo general, la población considera que los profesionales de la salud desarrollan una actitud empática con el paciente. Sin embargo, en la realidad peruana, muchos pacientes se quejan del trato que reciben por parte de algunos profesionales de la salud, lo cual nos hace tener en consideración evaluar la empatía desde el periodo de formación preprofesional. Debido a todo ello, es importante evaluar este aspecto en los estudiantes de pregrado, con la finalidad de indagar el nivel de empatía, y, de acuerdo a los resultados obtenidos, se puedan proponer estrategias de acción la formación de los estudiantes.

El estudio fue exploratorio y de corte transversal, y se rigió, desde el punto de vista bioético, por las normas de Helsinki. La población estuvo conformada por los estudiantes del primero al quinto ańo académico de la carrera de Obstetricia, que alcanza un total de 219 , equivalente al $60,33 \%$ de la población. Se utilizó como instrumento de investigación la Escala de Empatía Médica de Jefferson (EEMJ), que investiga tres factores: el cuidado con compasión, la toma de perspectiva y el ponerse en el lugar del otro. La aplicación fue anónima y confidencial. Antes de ser aplicado, 
el instrumento fue sometido a criterio de jueces (tres académicos relevantes de profesiones médico-gíneco-obstetra, obstetra y psicólogia), con el objeto de verificar la validez cultural y de contenido. Se realizó una prueba piloto para comprobar la comprensión de la escala por parte de los estudiantes, la que se adaptó culturalmente.

Sobre los resultados, el nivel empático se situó en un promedio de 105,95 puntos, de un nivel máximo de 140 puntos. Sin embargo, si consideramos que el potencial de crecimiento de los estudiantes (indicador que muestra el grado de avance de los niveles de empatía) de quinto año con respecto a los de primer ańo, fue cubierto solamente en un 10,13\%; esto significa que debemos reforzar el desarrollo de habilidades sociales e inteligencia emocional en el currículo de estudios desde los primeros años de formación, mediante diferentes actividades, como la realización de talleres que favorezcan el desarrollo de la empatía, o incorporar horas de prácticas a las asignaturas necesarias que permitan el mayor contacto directo de los estudiantes con la población, con énfasis en el trinomio madre-padre-nińo, especialmente en el área de la salud sexual y reproductiva, desde los primeros años de la carrera, entre otros aspectos. 